\title{
La música, un instrumento en la enseñanza del español como lengua extranjera mediante la aplicación de las nuevas tecnologías
}

\author{
RubÉn CRISTÓBAl Hornillos \\ IES José Martí, Varsovia \\ Juan de Dios Villanueva RoA \\ Universidad de Granada
}

Recibido: 1 abril 2012 / Aceptado: 18 enero 2013

ISSN: $1697-7467$

\begin{abstract}
RESUMEN: Este artículo expone una forma diferente de aprender español como lengua extranjera, partiendo de las canciones y con el uso de las Nuevas Tecnologías, con las videoconsolas y karaokes, analizando los intereses y necesidades del alumnado, sin que sea precisa una gran especialización en el mundo musical del profesor. Los diferentes aspectos del español como LE van apareciendo en los temas musicales, y a partir de ahí el alumno va formándose y avanzando en su proceso de aprendizaje con el trabajo de los temas que se sugieren y con las tecnologías más comunes en el mercado e Internet.

Palabras clave: Español como lengua extranjera, música y aprendizaje del español, lengua española y temas musicales
\end{abstract}

The music, a tool in teaching Spanish as a foreign language through the application of new technologies

\begin{abstract}
This article aims to describe an innovative way of learning Spanish as a foreign language (FL) based on the utilization of songs and ICTs. The initiative incorporates the use of video game consoles and karaoke and takes into account the interests and needs of learners without teachers being required to have in-depth knowledge of music. A number of different linguistic aspects of Spanish are treated in this project and students are able to train and develop their skills by working on the songs which are proposed in addition to using commonly employed ICTs and Internet.
\end{abstract}

Keywords: Spanish as a foreign language, music and learning Spanish, Spanish language and songs

\section{InTROduCCIÓN}

La música siempre acompañó a la persona, al individuo, tanto en sus expresiones sociales como en las religiosas. Fue vehículo para exteriorizar miedos y alegrías, ruegos y creencias. La lengua se hizo melodía, y los sonidos de los instrumentos acompasaron el mensaje desde un principio, fuesen aquellos un simple madero o un sofisticado violín.

Por otro lado, las nuevas tecnologías han venido a abrir campos nuevos, inimaginables hace apenas unas décadas, en el desarrollo de las expresiones humanas. La música no solo 
no podía quedarse atrás, sino que ha sido una de las grandes favorecidas por estas nuevas tecnologías.

La enseñanza ha venido utilizando la música como fin, pero también como medio de aprendizaje de ella misma y de otras disciplinas. En este artículo pretendemos acercar la música al aprendizaje de la segunda lengua, del español como lengua extranjera, con una metodología y actividades que vienen a aprovechar los nuevos elementos tecnológicos y las ventajas que las letras de las canciones y las canciones mismas tienen con respecto a nuestro fin. A través de este trabajo pretendemos crear un marco teórico y práctico que permita aprovechar las principales virtudes de los videojuegos, y más concretamente de los videojuegos musicales, para su explotación didáctica en la enseñanza de Español como Lengua Extranjera o como Segunda Lengua.

Tal como nos recuerda Esmeralda Jiménez (2006), Piaget ha demostrado en numerosos estudios la estrecha relación que existe entre la estructura mental y la actividad lúdica. "A través del juego se enfrenta el sujeto a nuevos problemas, buscando solucionarlos en un intento de reencontrar un equilibrio entre él mismo y el mundo que le rodea", señala. En este mismo sentido, numerosos investigadores, como recoge Alfonso Méndiz (2008) en una revisión crítica de la investigación sobre Videojuegos y Educación, han insistido en las habilidades que despiertan o ponen en valor los videojuegos y que son aprovechables para fines educativos, como la atención, la concentración, la creatividad o la resolución de problemas.

Los videojuegos musicales favorecen el desarrollo de actividades educativas más diversas que la simple utilización de canciones en clase, por lo que se enriquecerá el proceso de aprendizaje y se potenciará la participación del alumno y su autonomía, y esto gracias a su atracción, dinamismo e interactividad. Así mismo, los numerosos estímulos auditivos (sonido, música), visuales (fotografías, animación, vídeos e incluso imágenes tridimensionales) y escritos (como informaciones textuales) que entran en juego pueden servir como un extraordinario input para el aprendizaje de las destrezas que queremos mejorar en los alumnos, que son precisamente las destrezas orales, escritas y de interacción. Es decir, el alumno, que con toda probabilidad estará ya acostumbrado a utilizar de un modo lúdico los videojuegos y la música - bien de forma separada o conjunta en el caso de que esté familiarizado ya con los juegos musicales- tendrá la percepción de estar jugando, es decir, de estar divirtiéndose con su aprendizaje, lo que potenciará factores como la motivación, la atención o la concentración, que serán fundamentales para alcanzar nuestro objetivo principal, que es potenciar el aprendizaje del español.

Otras características que hacen de la canción una extraordinaria fuente de input son su brevedad y su autenticidad ya que, según analizan Santamaría (2000) y Ruiz (2005), se trata de textos completos cerrados con un "mensaje comunicativo concreto y coherente", además de un "léxico asociativo y concentrado". A estas ventajas del uso de la canción en clase, otros autores añaden -tal como recuerda Ruiz (2005)-, "la sencillez de los argumentos y estructuras de un lenguaje simple e informal" y el uso de "formas más próximas al lenguaje conversacional e informal".

De este modo, la selección de los textos de un modo apropiado a las particularidades del grupo meta al que va dirigido (edad, contexto social, temas preponderantes...) y del propio aula (nivel de español de los alumnos, aspecto lingüístico en el que se pretende incidir...), junto a la ventaja de trabajar cada canción de una forma dinámica e interactiva gracias a las nuevas tecnologías, incrementan aún más el valor que tiene la canción como recurso en la 
enseñanza de ELE. Evidentemente, el uso de canciones por sí solo no sirve para aprender una lengua, por lo que nuestro objetivo es analizar y facilitar su papel como una tarea más en el proceso de aprendizaje, ya sea usada con una mayor o menor frecuencia- dependiendo de las circunstancias concretas del aula- y siguiendo la metodología que proponemos.

Hemos de tener presente que la música es un elemento cultural y de ocio con gran presencia en nuestra sociedad, lo que permite al alumno prolongar el aprendizaje fuera del aula, mediante constantes fenómenos de feedback y retroalimentación al poder escuchar la misma canción que ha trabajado en el aula. Por otro lado, el carácter rítmico de la música favorece su memorización de una forma más motivadora, rápida y duradera, tal y como señala Martínez Sallés (2002).

Cassany (1994) dice que "Escuchar, aprender y cantar canciones en clase es una práctica de valor didáctico incalculable". Las razones que arguyen los expertos para defender el potencial didáctico de la música son múltiples y de carácter muy diverso. Algunas de estas razones son de índole comunicativo, tanto porque permiten trabajar al mismo tiempo las distintas destrezas orales, auditivas y escritas, como por la autenticidad que presentan las letras de las canciones cuando estas son elegidas en función del grupo meta con el que se van a utilizar. Otras son de índole lingüística, porque además de ofrecer una amplia gama de registros y estilos, las canciones suelen utilizar estructuras simples y repetidas, lo que permite trabajar de forma selectiva distintos aspectos gramaticales, sintácticos, léxicos y prosódicos (tales como el acento, los tonos y la entonación) de una forma integral.

Pero el uso de la música en la enseñanza de una lengua también cuenta con importantes argumentos conceptuales, culturales y emocionales, porque cada canción como unidad de trabajo representa en sí misma una concepción distinta de la realidad, sujeta a distintas interpretaciones, y transmite una carga cultural y emocional a los alumnos que pueden servirle como verdaderos input que desencadenen un proceso de aprendizaje significativo.

Señala Rosana Larraz (2008) que "Las canciones contienen lengua auténtica, proporcionan vocabulario y gramática, permiten trabajar la pronunciación y favorecen tanto el conocimiento de los aspectos culturales de la lengua de estudio, como la asociación de la lengua a la cultura". A todo esto, añade, "el poder de la música para estimular las emociones, la sensibilidad y la imaginación sin olvidar las consecuencias que se derivan de la capacidad que poseen las canciones para engancharse a nuestra memoria". Numerosos expertos coinciden en que a pesar de los indudables beneficios de la música para la enseñanza de idiomas y a su larga tradición en el aula, todavía no se han explotado las posibilidades de esta herramienta en el aula, y no ha sido así porque, según adelantaba Javier Santos (1996), "ni las editoriales ni la discográficas ni la industria de los videojuegos han secundado el interés creciente que comparten muchos profesores y la mayoría de los alumnos". De esta forma, la principal renovación o revolución en este campo ha venido dada por un lado de la mano de los propios docentes, que han ido desarrollando diferentes actividades didácticas para trabajar aspectos lingüísticos y comunicativos concretos; y por otro de la mano de Internet, lo que les ha permitido disponer de un abanico prácticamente ilimitado de temas $\mathrm{y}$, al mismo tiempo, compartir sus actividades didácticas y sus investigaciones con el resto de los docentes.

Esta nueva realidad tecnológica ha abierto nuevos caminos y formas de trabajo en la enseñaza de idiomas en general, donde contamos con múltiples actividades y juegos interactivos desarrollados para ejercitar la gramática, la sintaxis, el vocabulario o incluso la fonética, 
como podemos ver en propuestas y estudios, como los de Long (2005), Higueras (2004) y Adell (2004). Sin embargo, en la mayoría de los casos todavía no se aprovechan las posibilidades interactivas que ofrecen no solo los ordenadores, sino también las videoconsolas y otras plataformas desarrolladas principalmente para el mercado del ocio. Quizá esto se deba a que la música se considera ya de por sí un estímulo suficientemente motivador para el alumno, pero ello no debe llevarnos a renunciar a aprovechar las múltiples posibilidades que ofrecen las nuevas tecnologías en su aplicación a la enseñanza de ELE a través de la música.

\section{Hacia Un Enfoque QUe integre el USO de las TIC}

Pese al importante camino realizado y a los avances constatados en los últimos años, la mayor parte del material existente en la enseñanza del español como segunda lengua sigue sin aprovechar las posibilidades que ofrecen las nuevas tecnologías. En general, todo este material solo se sirve del ordenador e Internet para escuchar, por un lado, las canciones sobre las que debe trabajar y, por otro, aunque de forma independiente, para realizar las actividades preparadas sobre estas. Así, la mayoría de estas propuestas no aprovechan las posibilidades interactivas que presentan el ordenador ni mucho menos otras plataformas interactivas como las videoconsolas.

Además, la mayor parte de las actividades existentes están diseñadas para canciones concretas, de modo que apenas existen métodos generales que se puedan aplicar a distintos temas y que partan, como se recoge en este trabajo, del Marco Común Europeo de Referencia.

\section{LOS VIDEOJUEGOS Y SU APLICACIÓN EDUCATIVA}

Solo en España, según recoge una Proposición no de Ley aprobada por el Congreso de los Diputados el 25 de marzo del año 2010, "la industria del videojuego factura anualmente más de 700 millones de euros -una cifra que se disparó hasta los 1.432 millones de euros en ese mismo año según los datos que maneja el sector-, y emplea directamente a más de 3.000 personas", lo que nos convierte en el cuarto país europeo, y el sexto mundial, en consumo de software de entretenimiento interactivo. De hecho, en la actualidad, además de mover una ingente cantidad de dinero e intereses, los videojuegos han modificado la forma de relacionarse de muchas personas, sus hábitos y, en definitiva, su modo de vida.

Unos estímulos que además de explicar la evolución que han tenido los videojuegos en el aspecto lúdico, tienen a su vez un extraordinario potencial en el campo educativo, ya que pueden convertirse en input que favorezcan prácticamente cualquier proceso de aprendizaje. A eso hay que añadir que la extraordinaria implantación lúdica que tiene el videojuego entre el sector infantil favorece también una rápida aceptación de su uso en el aula por parte de los alumnos, quizá mayor incluso que la que cuenta entre los propios profesores.

Como resultado de sus experiencias desarrolladas, la profesora de Psicología de la Educación Pilar Lacasa (2011) ha llegado a la conclusión de que el videojuego puede ser un instrumento educativo de primer orden, y que este puede contribuir más que otros medios a que las personas lleguen a participar responsable y críticamente en una cultura digital, 
ya que exigen poner en práctica habilidades propiamente humanas, por ejemplo, dialogar, argumentar y crear.

Un proyecto pionero es <e-Adventure $>$, una plataforma de autoría de juegos educativos y entornos de simulación 2D desarrollada por el grupo de investigación $<\mathrm{e}-\mathrm{UCM}>$ del Departamento de Ingeniería del Software e Inteligencia Artificial de la Facultad de Informática de la Universidad Complutense de Madrid con el objetivo de introducir videojuegos educativos en el flujo de clase. Para conseguirlo, este programa pretende reducir los costes de producción de videojuegos y permitir su desarrollo sin necesidad de conocimientos de programación, con el fin de que los profesores se acerquen al proceso de creación de juegos y puedan dotarlos de un valor pedagógico real. Con este mismo objetivo, los juegos desarrollados pueden ser modificados de manera sencilla para que los educadores puedan amoldarlos a las necesidades específicas de su clase.

\section{Los VIDEOJUEgos MUSICALES}

Los videojuegos musicales, más conocidos como videojuegos de karaoke, han tenido una evolución fulgurante en el mercado del entretenimiento durante los últimos años propiciada fundamentalmente por el desarrollo tecnológico y el éxito social de las videoconsolas. Esto ha permitido experimentar con aplicaciones de ocio sociales dirigidas principalmente a entornos familiares y grupos de amigos, como alternativa al videojuego de uso individual.

Los videojuegos de karaoke son aquellas aplicaciones destinadas a videoconsolas y ordenadores, y cuya jugabilidad está orientada significativamente a que el jugador ponga a prueba su habilidad para cantar y entonar. Frente a los karaokes convencionales, estos videojuegos cuentan con nuevas funciones, como la posibilidad de escuchar las canciones con la voz original del cantante y superponer la voz de los participantes, recibir valoraciones o concursar entre varios usuarios en distintos modos. Dado el carácter lúdico pero también social de la música, estos juegos están orientados principalmente a grupos de dos o más personas, aunque también pueden ser utilizados por jugadores de forma individual con el fin de aprenderse una canción, perfeccionar su voz y su entonación o, simplemente, pasar un rato entretenido.

\section{Propuesta metodológica}

La propuesta metodológica que presentamos en los siguientes apartados parte del modelo tradicional estructuralista, en el que ya se utilizaban canciones y poesías en el aula como unidades de trabajo para estudiar o reforzar uno o varios aspectos lingüísticos concretos (básicamente vocabulario, estructuras sintácticas y gramaticales y comprensión auditiva), pero lo hace con un enfoque renovado que se enmarca más en el modelo comunicativo y en el enfoque por tareas, aunque su identificación como tal puede ser cuestionable porque todas la tareas se resumen en una: que el alumno cante.

La diferencia en cada una de estas tareas son los contenidos y las destrezas que trabaja el alumno durante todo el proceso de aprendizaje, ya que lo que menos importa, lógica- 
mente, es su calidad artística, siendo fundamental el desarrollo de sus destrezas lingüísticas. Según apuntan varios investigadores, como Santamaría, R. (2000), la canción se convierte de este modo en un pretexto para su aprendizaje, pero en un pretexto significativo, porque, además de servir como hilo temático de todas las subtareas, el ritmo y la música favorecen la memorización y la interiorización del lenguaje, es decir, la comprensión y la producción.

El otro gran aliado para el desarrollo de cada tarea serán las nuevas tecnologías, principalmente las aplicaciones disponibles para videoconsolas, porque envía al alumno constantes feedback correctivos que favorecen la interacción y la retroalimentación durante el proceso como sucede, por ejemplo, al escuchar la canción con o sin letra, al recibir una valoración del progreso, al volver a escuchar su voz comparándola con la letra original o a "competir" con otro compañero, entre otras actividades posibles

Igualmente, tanto algunas de las características propias de las canciones ya señaladas (la autenticidad, su cercanía al lenguaje convencional o su valor estético y social...), como el carácter lúdico y actual de los videojuegos, servirán para motivar al alumno en la realización de las tareas previas y derivadas, por usar la terminología que propone Martín Peris (2004), para redefinir las tareas posibilitadoras y capacitadoras, unos términos que el autor considera insuficientes para referirse a las fases que componen el proceso de aprendizaje por tareas.

\section{LA REFERENCIA DEL MARCO COMÚN EUROPEO}

El Marco Común Europeo de Referencia (MCER) para las Lenguas señala que "todas las tareas, sean de la clase que sean, requieren la activación de una serie de competencias generales adecuadas, como por ejemplo: el conocimiento y la experiencia del mundo, el conocimiento sociocultural, destrezas tales como las interculturales, las de aprendizaje y las sociales y conocimientos prácticos habituales de la vida cotidiana".

Entre las tareas y propósitos comunicativos recomendables en el aula, este documento recoge la actividad de cantar (refiriéndose expresamente a canciones de música pop, canciones infantiles y canciones populares), tanto al tratar el uso estético de la lengua como en el apartado de las actividades de expresión oral. A pesar de ello, el Marco Común Europeo de Referencia para las Lenguas no recoge descriptores relacionados con la actividad propiamente dicha de cantar, algo que es comprensible por tratarse de un uso comunicativo excesivamente específico. No obstante, el propósito de nuestra tarea no será que el alumno aprenda a cantar, sino que mejore sus destrezas lingüísticas y adquiera unas competencias y unos contenidos lingüísticos, por lo que una vez elegida una canción para un nivel y un caso concreto, el profesor (o el propio alumno en el caso del autoaprendizaje), podrá desarrollar las tareas que considere apropiadas siguiendo los descriptores del MCER sin necesidad de tener conocimientos sobre la materia, ya que hemos partido precisamente de estos descriptores para clasificar las canciones por niveles y para proponer los contenidos y competencias para cada caso.

En cuanto a las destrezas lingüísticas, aunque muchas de las propuestas didácticas tradicionales con canciones se centran en potenciar la comprensión auditiva, lo cierto es que el trabajo con una canción — como sucede casi con cualquier texto-, nos permite también potenciar la comprensión escrita — por ejemplo mediante la lectura de las letras y documentación al respecto-, la expresión oral — principalmente la pronunciación-y la expresión 
escrita - por medio de tareas previas o derivadas planificadas para este efecto-, sin olvidar la interacción oral y escrita, que también se puede practicar gracias al trabajo en grupo y a las opciones interactivas que ponen a nuestra disposición las nuevas tecnologías. De este modo, por medio de la explotación de las canciones se puede integrar el trabajo de las destrezas con cualquier tipo de contenido lingüístico (fonéticos, morfológicos, gramaticales, léxicos o sintácticos) y de competencias socioculturales y comunicativas.

Una de las propuestas más novedosas en este sentido es Encantados, publicado en diciembre de 2010 por la Subdirección General de Cooperación Internacional del Ministerio de Educación de forma coordinada con diversos institutos de español en el extranjero. En ella, se parte de un total de quince canciones para proponer diversas tareas o actividades relacionadas directamente con las destrezas lingüísticas y los contenidos que se pretenden trabajar, tomando también como punto de partida el Marco Común Europeo de Referencia, para convertirla en una herramienta al servicio del aprendizaje del español.

Pero una de las diferencias principales de nuestra propuesta frente a esta última, además de la utilización de las videoconsolas como elemento trascendental, es que en este trabajo hemos renunciado a desarrollar una tarea completamente diferenciada para cada canción, porque ello limita por un lado la disponibilidad y elección final de los temas e impide, por otro lado, que cada tarea esté adaptada realmente a las características del grupo meta, las necesidades del aula concreta o los contenidos y destrezas que se quieren trabajar de una forma más intensa.

A cambio, esta propuesta consta de una plantilla general que incluye una serie de subtareas adaptables para que el profesor o el alumno autodidacta puedan escoger y desarrollar aquellas que considere más acordes a las competencias y los contenidos que quiere reforzar. Con el objetivo de facilitar esta adaptación, hemos elaborado una extensa relación de temas disponibles en español (230) para las distintas aplicaciones y plataformas referenciados por nivel, con algunos de los aspectos más interesantes que se pueden trabajar con cada una de las canciones (no se adjunta esta relación por las características propias de este medio). Finalmente, también hemos elaborado una relación con las principales videoconsolas que hay en el mercado y con las aplicaciones y opciones interactivas disponibles para dichas plataformas que van a ser útiles para llevar a cabo las tareas propuestas. Al igual que con los temas musicales, no podemos incluir esta relación en este trabajo por el espacio que ocupa.

\section{Material didáctico}

Si uno de los principales objetivos de este trabajo es crear una herramienta útil y flexible al servicio de la Enseñanza de Español como Lengua Extranjera, es necesario que esta resulte principalmente ágil desde el punto de vista práctico sin dejar de ser por ello rigurosa desde un punto de vista teórico. Con la vista puesta en este doble objetivo, la presentación del material didáctico propiamente dicho se compone de dos partes claramente diferenciadas que, una vez combinadas, permitirán al alumno desarrollar la tarea que mejor se adapte a sus circunstancias y necesidades a partir de un proceso sencillo tanto para el profesor de un aula de español como para el alumno en su proceso de autoaprendizaje.

Se analizan las plataformas tecnológicas y sus características principales aplicables a nuestro objetivo. Como ya hemos indicado, las características de esta publicación nos im- 
piden adjuntar los temas, con los que el profesor o alumno podría consultar (ordenados por autor o intérprete) y seleccionar el que mejor se adaptase a sus circunstancias de aprendizaje, así como a otros factores, como sus gustos personales, su contexto sociocultural, las situaciones comunicativas prioritarias o su disponibilidad, en función de las plataformas y aplicaciones a las que tiene más fácil acceso. Hemos desarrollado una secuencia didáctica de tareas organizada en cuatro fases (planteamiento, preparación, ejecución y post-tarea) que, en última instancia, se presentará como una plantilla de trabajo para que el profesor o el alumno autodidacta escoja y desarrolle la que mejor se adapta a sus circunstancias y a la canción con la que va a trabajar.

De este modo, desde el punto de vista práctico, el material elaborado se comporta a modo de una matriz con distintas entradas, para que en función de cada caso concreto (contexto sociocultural, grupo meta, nivel de aprendizaje, preferencias, destrezas y contenidos que se desean trabajar...) pueda obtenerse un resultado diferenciado, es decir, una tarea personalizada.

\section{Canciones, aplicaciones y plataformas}

El trabajo desarrollado consta de dos tablas, una de las cuales (Apéndice I) desglosa las características y funciones que ofrecen las principales aplicaciones o juegos tipo "karaoke" disponibles para consolas y para ordenador; mientras que la otra (que por las razones expuestas anteriormente no puede aparecer aquí) contiene un listado de 230 temas en español disponibles para las distintas plataformas y que hemos seleccionado siguiendo tanto criterios de reconocimiento, como por las posibilidades de implementación que tienen, desde nuestro punto de vista, para la enseñanza de Español como Lengua Extranjera. Para facilitar la elección por parte del usuario final, hemos ordenado los temas por artista, señalando la época y el género al que pertenecen, y hemos incluido un apartado donde se destacan los contenidos y competencias que se pueden trabajar en cada uno de los temas -sin menoscabo de otros que se puedan añadir- referenciados al nivel para el que estaría indicado cada uno de ellos según los descriptores del Marco Común Europeo de Referencia. De este modo, el usuario final pueda cruzar datos y comprobar con solo un golpe de vista las posibilidades que le ofrece cada aplicación o plataforma, así como las principales características de los temas disponibles en cada una de estas.

Además de las aplicaciones disponibles propiamente para videoconsolas, hemos incluido a modo de ejemplo un equipo básico de karaoke convencional y dos aplicaciones para ordenador. La primera de estas es un programa informático de karaoke, VanBasco's Karaoke Player, que se puede descargar de forma gratuita y utilizar en el ordenador, y otra aplicación vía Internet, www.redkaraoke.es, en los que se reducen algunos modos y opciones de interactividad, pero que pueden ser una solución válida para quien no disponga de otra plataforma o desee trabajar con algún tema específico que no esté disponible actualmente para estas.

\section{Secuencia didáctica de la plantilla}

En este punto analizamos la secuencia didáctica y las principales características de cada una de las fases en las que se divide la plantilla base de trabajo que hemos desarrollado partiendo de las directrices recogidas por el Marco Común Europeo de las Lenguas para 
que sean aplicables a las distintas canciones o "unidades de trabajo", así como a la mayor parte de las funciones disponibles en las plataformas y aplicaciones analizadas.

La plantilla base se divide en cuatro fases: planteamiento, preparación, ejecución, post-tarea -siguiendo la terminología propuesta por Martín Peris (2004)-, cada una de las cuales recoge una serie de subtareas diseñadas de forma abierta y flexibles para que puedan adecuarse a cada caso. De este modo, el profesor o el alumno autodidacta podrá elegir las tareas que mejor se adapten a sus circunstancias y desarrollarlas o combinarlas de una forma flexible dependiendo de aspectos como el grupo meta, su contexto sociocultural, el nivel de aprendizaje, las preferencias personales o las destrezas y contenidos lingüísticos en los que se desea hacer hincapié, ya sean de carácter léxico, fonético, morfológico, gramatical, sintáctico o comunicativo.

Las subtareas propuestas durante la fase de planteamiento son principalmente tareas nociofuncionales relacionadas con el contexto sociocultural de la canción y su autor, que están orientadas a que el alumno busque, comprenda, redacte o exponga información -entre otras actividades- sobre el tema que se va a trabajar posteriormente. De esta forma, el alumno podrá comenzar a poner en práctica sus destrezas de comprensión y expresión escrita -básicamente mediante la lectura y elaboración de documentos- o la comprensión auditiva -cuando algunos de los recopilados sean audios o vídeos-, además de la interacción y expresión oral en la presentación de sus conclusiones o en el debate con sus compañeros.

Durante la fase de preparación se desarrollan lo que venían denominándose tareas posibilitadoras o capacitadoras, pero que Martín Peris (2004) prefiere llamar tareas previas, por considerar que no solo están destinadas a la consecución de la tarea final, sino a que el alumno aprenda durante su realización. Así, se incluyen tareas relacionadas directamente con la situación comunicativa generada durante el acto de cantar, aunque fundamentalmente con el rol del oyente. Es decir, algunas de estas subtareas consistirán en leer la letra de la canción, escucharla varias veces con y sin subtítulos, buscar el vocabulario desconocido, trabajar algunas expresiones lingüísticas, hacer una primera exposición o debate sobre el tema o la idea general de la canción, analizar aspectos socioculturales o comunicativos que podemos identificar en el texto como manifestación artística, etc. De esta forma, la canción sirve como input o punto de partida para el trabajo de contenidos lingüísticos y el desarrollo de destrezas de compresión auditiva y escrita, al principio, pero también de producción e interacción después.

A diferencia de la anterior, la fase de ejecución se centra en el output, es decir, en conseguir que los alumnos produzcan un mensaje lingüístico, en este caso, que canten. Lejos de cualquier aspiración artística, la música y el ritmo -la canción en general- son un pretexto para inducir a los alumnos a producir un producto lingüístico oral, es decir un output controlado y coherente. Para ello, el trabajo con videoconsolas nos permite contar con un sistema automático de feedback correctivo, ya que estas aplicaciones poseen mecanismos de valoración y retroalimentación sobre el acierto de los jugadores, que aunque está planificado para valorar la supuesta capacidad musical del participante, podemos adaptarlo a nuestro objetivo. Además, también podemos aprovechar con este fin otras opciones que presentan las videoconsolas, como las canciones a dúo, los "combates", las canciones en grupo, etc., que permiten participar al mismo tiempo a varios alumnos. De este modo podemos motivarlos, como si de un juego se tratara, a mejorar su pronunciación, corrección y fluidez; usar el vocabulario adecuado (tanto respecto a la forma como al significado), dar el sentido 
correcto a cada expresión, articular las oraciones de forma apropiada al contexto, ponerse en el lugar de esta situación comunicativa y, en definitiva, comprender y reconstruir casi de forma inconsciente y automática el mensaje que ha escuchado previamente.

Y por último, llegamos a la fase post-tarea, en la que el objetivo principal de las tareas derivadas es que el alumno produzca, a partir del mismo input, un output menos controlado y repetitivo que en la fase anterior, es decir, un producto lingüístico más creativo y en el que podemos corregir aquellos errores que presente el alumno al trabajar en una situación de mayor libertad y, por tanto, de mayor inseguridad lingüística. Para conseguirlo, el profesor -o el propio alumno en su caso- debe procurarse una atmósfera de trabajo que favorezca la motivación, el esfuerzo y la certidumbre sobre los objetivos y los resultados de las tareas a desarrollar, que estarán directamente vinculadas con las que ya se han realizado en las fases anteriores. Así, algunas de estas tareas derivadas que se proponen en la plantilla van orientadas a que el alumno resuma la idea principal de la canción, analice el propio proceso creativo del artista al crear o interpretar esa canción, sitúe esa producción artística en un ámbito personal, un contexto social o una tradición cultural, exprese su opinión y conjeture sobre el proceso de creación y el producto final, o cree otros productos lingüísticos a partir de este, como sería inventar una historia alternativa que encaje con el videoclip. En definitiva, tareas que permitan al alumno apropiarse de esta situación comunicativa para producir, como resultado de sus circunstancias individuales, un mensaje lingüístico propio.

\section{Conclusiones}

La música en español es un potente atractivo para los hablantes de otras lenguas como pone de manifiesto la gran difusión que han tenido y siguen teniendo cantantes y canciones en español en todo el mundo. De hecho, son muchas las personas que se introducen en nuestra lengua por primera vez precisamente a través de las letras de las canciones, aunque muchas veces no exista todavía un propósito real de aprender el español.

La principal conclusión es que los juegos tipo karaoke diseñados para las videoconsolas con fines lúdicos nos ofrecen interesantes posibilidades para aprovechar las sinergias que suman las nuevas tecnologías y la música en su aplicación a la enseñanza de ELE. La interactividad de las plataformas tecnológicas proporciona una serie de mecanismos de retroalimentación o feedback extraordinariamente útiles y motivadores para el desarrollo de actividades de aprendizaje de una lengua extranjera con canciones, especialmente cuando se produce en situaciones de autoaprendizaje parciales o totales. Así mismo, cuando el aprendizaje se desarrolla bajo la tutela de un profesor, el papel de este puede resultar trascendental tanto para desarrollar y adaptar las tareas de una forma adecuada y coherente, así como para motivar, orientar, dirigir y corregir a los alumnos durante todo el proceso posterior con el objetivo de que este se resuelva con las máximas garantías de éxito.

Tanto para unos casos como para otros, a lo largo de este trabajo se han presentado unas pautas de trabajo que permiten adaptar las numerosas posibilidades de estos videojuegos para nuestro propósito educativo. Esto nos lleva a concluir recordando el interés que tendría el desarrollo de aplicaciones educativas para la videoconsolas y, sobre todo, para otras plataformas que cuentan con una mayor implementación en el aula, como es el caso de las pizarras digitales (PDi). 
Queda ahí como propuesta de trabajo para uno de los múltiples caminos de exploración que nos ofrece actualmente la sinergia entre la educación y las Tecnologías de la Información y la Comunicación (TICs).

\section{REFERENCIAS BIBLIOGRÁFICAS}

Adell, J. (2004). "Internet en el aula: las WebQuest Edutec", Revista Electrónica de Tecnología Educativa, 17.

Cassany, D., Luna, M. y Sanz, G. (1994). Enseñar lengua. Barcelona: Graó.

García García, F. (Director de la colección de la Serie Informes) (2004). Videojuegos y educación. Madrid: CNICE, Serie Informes. En CD ROM y en Internet, disponible en: http:// ares.cnice.mec.es/informes/02/documentos/indice.htm, accedido el 30 de marzo de 2012.

Higueras, M. (2004). "Internet en la enseñanza del español”, en J..Sánchez e I. Santos (dirs.), Vademécum para la formación de Profesores. Enseñar Español como Segunda Lengua (L2) / Lengua extranjera (LE). Madrid: SGEL, 1061-1087.

Jiménez Rodríguez, E. (2006). "La Importancia del Juego", en $I+E$, Revista digital Investigación y Educación, 26.

Lacasa, P. (2011). Los videojuegos. Aprender en Mundos Reales y Virtuales. Madrid: Editorial Morata.

Larraz, R. (2008). "Canciones para enseñar lenguas", en Cuaderno Intercultural: disponible en: http://www.cuadernointercultural.com/canciones-para-aprender-idiomas accedido el 10 noviembre de 2011.

Long, M. (2005). “Adquisición de segundas lenguas (ASL) y nuevas tecnologías”, en Biblioteca Virtual redELE, número especial, mayo.

Martínez Sallés, M. (2002). Tareas que Suenan Bien. El Uso de Canciones en Clase de ELE. Bruselas: Consejería de Educación en Bélgica, Países Bajos y Luxemburgo.

Méndiz, J, y otros (2008). "Videojuegos y educación: una revisión crítica de la investigación y la reflexión sobre la materia", en Videojuegos y Educación. Grupo de Investigación sobre Videojuegos de la Universidad de Málaga. MEC.

Martín Peris, E. (2004). "Qué significa trabajar con tareas", en REDELE, disponible en http://www. educacion.gob.es/dctm/redele/Material-RedEle/Revista/2004_00/2004_redELE_0_18Martin. pdf?documentId=0901e72b80e0c9e3, accedido el 30 de noviembre de 2011.

Ruiz García, R. (2005). "De las baúles de la Piquer a las maracas de Machín. La canción como contenido cultural en la clase de ele", en REDELE. Biblioteca virtual $\mathrm{n}^{\circ} 3$.

Santos, J. (1996). "Música española contemporánea en el aula de español”, en Tendencias actuales en la enseñanza de español como lengua extranjera. Actas del VI Congreso Internacional de ASELE, Universidad de León.

Santos, J. (1996). "De la música contemporánea a la didáctica de E/LE”, en Didáctica de la Lengua y la Literatura para una sociedad plurilingüe del siglo XXI, Universidad de Barcelona.

Santamaría, R. (2000). "Del poder evocador de la poesía al ritmo de la música en el aula de ELE”, en Frecuencia L, Madrid, 5. 
Apéndice I: Tabla de funciones por aplicaciones y plataformas

\begin{tabular}{|c|c|c|c|c|c|c|c|c|c|}
\hline & 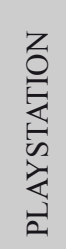 & 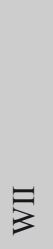 & $\begin{array}{l}0 \\
0 \\
m \\
x \\
0 \\
0 \\
x\end{array}$ & \multicolumn{2}{|c|}{ 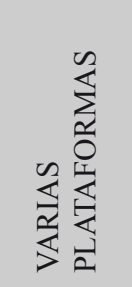 } & 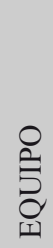 & 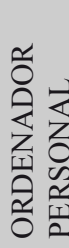 & 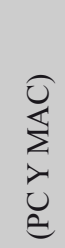 & \\
\hline \begin{tabular}{|c} 
OPCIONES \\
DE LOS \\
VIDEOJUEGOS \\
CON \\
APLICACIONES \\
EDUCATIVAS
\end{tabular} & 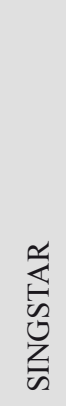 & $\begin{array}{l}0 \\
Z \\
心 \\
D\end{array}$ & 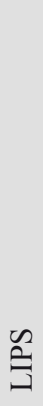 & $\begin{array}{l}\qquad \\
\vdots \\
\text { 光 }\end{array}$ & 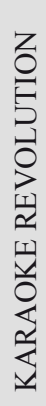 & 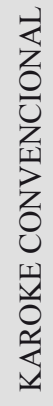 & 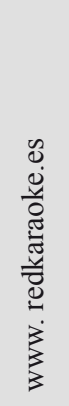 & 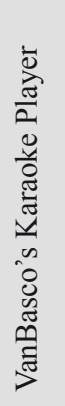 & $\begin{array}{c}\text { DESTREZAS } \\
\text { QUE PUEDEN } \\
\text { REFORZARSE EN } \\
\text { SU APLICACIÓN } \\
\text { PARA EL } \\
\text { APRENDIZAJE DE } \\
\text { ELE }\end{array}$ \\
\hline $\begin{array}{l}\text { Modo solo letra } \\
\text { (o karaoke sin } \\
\text { sonido) }\end{array}$ & $\mathrm{X}$ & $\mathrm{X}$ & $X$ & $\mathrm{X}$ & $X$ & $\mathrm{X}$ & $\mathrm{X}$ & $\mathrm{X}$ & Comprensión escrita \\
\hline $\begin{array}{l}\text { Karaoke } \\
\text { convencional } \\
\text { (escuchar música } \\
\text { con subtítulos) }\end{array}$ & $X$ & $X$ & $X$ & $X$ & $X$ & $X$ & $\mathrm{X}$ & $\mathrm{X}$ & $\begin{array}{l}\text { Comprensión } \\
\text { auditiva y escrita }\end{array}$ \\
\hline $\begin{array}{l}\text { Karaoke con la } \\
\text { voz original del } \\
\text { cantante y la del } \\
\text { participante }\end{array}$ & $\mathrm{X}^{10}$ & $X$ & $X$ & $X$ & $X$ & - & - & - & $\begin{array}{l}\text { Comprensión } \\
\text { auditiva y expresión } \\
\text { oral }\end{array}$ \\
\hline $\begin{array}{l}\text { Jukebox o } \\
\text { gramola (escuchar } \\
\text { la canción } \\
\text { original, ver } \\
\text { videoclip y cantar } \\
\text { sin subtítulos) }\end{array}$ & $\mathrm{X}$ & $\mathrm{X}$ & $\mathrm{X}$ & $\mathrm{X}$ & $\mathrm{X}$ & 一 & $\mathrm{X}$ & - & $\begin{array}{l}\text { Comprensión } \\
\text { auditiva }\end{array}$ \\
\hline $\begin{array}{l}\text { Versión corta de la } \\
\text { canción }\end{array}$ & X & $X$ & $X$ & $X$ & $X$ & 一 & - & $X$ & $\begin{array}{l}\text { Comprensión } \\
\text { auditiva y escrita }\end{array}$ \\
\hline
\end{tabular}




\begin{tabular}{|c|c|c|c|c|c|c|c|c|c|}
\hline Modo pause & $\mathrm{X}$ & $\mathrm{X}$ & $\mathrm{X}$ & $\mathrm{X}$ & $X$ & $\mathrm{X}$ & $\mathrm{X}$ & $\mathrm{X}$ & \\
\hline \begin{tabular}{|l} 
Modo \\
instrumental para \\
cantar en karaoke \\
con subtítulos y \\
música (sin la voz \\
original)
\end{tabular} & $\mathrm{X}$ & $\mathrm{X}$ & $\mathrm{X}$ & $\mathrm{X}$ & $X$ & $\mathrm{X}$ & $\mathrm{X}$ & $\mathrm{X}$ & Expresión oral \\
\hline $\begin{array}{l}\text { Cantar la canción } \\
\text { con barras tonales } \\
\text { y con valoración }\end{array}$ & $\mathrm{X}$ & $\mathrm{X}$ & $\mathrm{X}$ & $\mathrm{X}$ & $X$ & - & - & - & Expresión oral \\
\hline $\begin{array}{l}\text { Cantar la canción } \\
\text { en modo dúo o } \\
\text { dueto }\end{array}$ & $\mathrm{X}$ & $\mathrm{X}$ & $\mathrm{X}$ & $\mathrm{X}$ & $\mathrm{X}$ & $\mathrm{X}$ & - & - & $\begin{array}{l}\text { Compresión } \\
\text { auditiva y escrita. } \\
\text { Expresión e } \\
\text { interacción oral }\end{array}$ \\
\hline $\begin{array}{l}\text { Cantar la canción } \\
\text { en modo duelo, } \\
\text { versus, batalla o } \\
\text { combate }\end{array}$ & $\mathrm{X}$ & $\mathrm{X}$ & $X$ & $\mathrm{X}$ & $\mathrm{X}$ & - & - & - & $\begin{array}{l}\text { Compresión } \\
\text { auditiva y escrita. } \\
\text { Expresión e } \\
\text { interacción oral }\end{array}$ \\
\hline $\begin{array}{l}\text { Cantar la } \\
\text { canción en modo } \\
\text { multijugador, pasa } \\
\text { el micro o Fiesta }\end{array}$ & $\mathrm{X}$ & $\mathrm{X}$ & 一 & $\mathrm{X}$ & $X$ & $\mathrm{X}$ & - & - & $\begin{array}{l}\text { Compresión } \\
\text { auditiva y escrita. } \\
\text { Expresión e } \\
\text { interacción oral }\end{array}$ \\
\hline $\begin{array}{l}\text { Playback } \\
\text { (reescuchar la voz } \\
\text { del jugador) }\end{array}$ & $\mathrm{X}$ & $\mathrm{X}$ & $\mathrm{X}$ & $\mathrm{X}$ & $\mathrm{X}$ & $\mathrm{X}$ & $\mathrm{X}$ & $\mathrm{X}$ & $\begin{array}{l}\text { Comprensión } \\
\text { auditiva }\end{array}$ \\
\hline $\begin{array}{l}\text { Playback de forma } \\
\text { simultánea a la } \\
\text { original (permite } \\
\text { comparar) }\end{array}$ & $\mathrm{X}$ & $\mathrm{X}$ & $\mathrm{X}$ & $\mathrm{X}$ & $\mathrm{X}$ & - & - & - & $\begin{array}{l}\text { Comprensión } \\
\text { auditiva }\end{array}$ \\
\hline
\end{tabular}

